\title{
Terminological Default Reasoning about Spatial Information: A First Step
}

\author{
Ralf Möller and Michael Wessel \\ University of Hamburg, Computer Science Department \\ Vogt-Koelln-Str. 30, 22527 Hamburg, Germany \\ \{moeller,mwessel\}@informatik. uni-hamburg.de \\ http://kogs-www. informatik. uni-hamburg.de/ $\{$ moeller,mwessel $\}$
}

\begin{abstract}
We extend the theory about terminological default reasoning by using a logical base language that can represent spatioterminological phenomena. Based on this description logic language called $\mathcal{A L C} \mathcal{R} \mathcal{P}\left(\mathcal{S}_{2}\right)$, which is briefly introduced, we discuss algorithms for computing so-called extensions ("possible worlds") of a world description and a set of defaults. We conclude with an application of the theory to problems in visual query systems and demonstrate the significance of the theory for spatioterminological reasoning in general and spatioterminological default reasoning in particular.
\end{abstract}

\section{Introduction}

For accessing spatial databases or geographic information systems (GIS), different query specification techniques have been proposed. For instance, the visual spatial query system VISCO developed in our group [9] can be used to query a spatial database (GIS) in a visual way. In contrast to conventional textual query systems the user is not required to learn a complicated textual query language in order to effectively use an information system. Users can query the database by drawing diagrammatic representations of what is to be retrieved from the spatial information system. However, experiences with the current VISCO system indicate that in the context of VISCO (and query systems in general), the specification of queries in a GIS still could be made easier by advances in research areas combining spatial and terminological reasoning.

First of all, the process of formulating (visual) queries can be facilitated by automatically completing queries in a meaningful way, therefore reducing the number of mouse interactions or -in the case of textual query languages- simplifying the composition of textual query elements. For instance, the process of selecting semantic concept descriptors for objects involved in a query (e.g. city, lake, country) can partly be automated by interpreting a partially specified query. In its current development stage, VISCO users can select concept descriptors from a list of over 300 predefined concepts. Thus, even a situationadapted reduction of the complete list of possibilities to a suitable subset or an order relation for sorting groups of possible concept canditates would be very appropriate. 


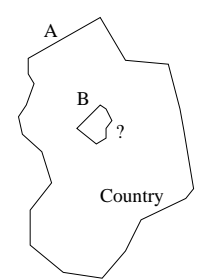

(a) Incomplete Query

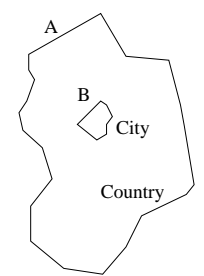

(b) Possibility 1

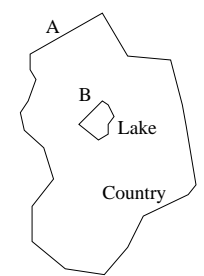

(c) Possibility 2

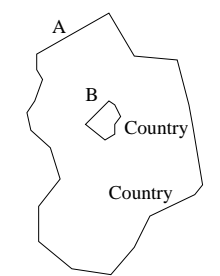

(d) Inconsistency

Fig. 1. Automatic completion of visual queries by application of default rules.

The goal of this paper is to present solutions for representing domain knowledge concerning spatial as well as terminological reasoning for interpreting spatial structures (e.g. visual queries). Intuitively speaking, our solution to the specific query completion problems is to model so-called default knowledge that is used to make queries more precise if it can be applied in a consistent way. We extend an algorithm for computing possible worlds from default rule applications and discuss under which conditions this algorithm can be applied, i.e. under which conditions default reasoning can be supported. In order to analyze the modeling problems in this context, we begin with a more detailed discussion of the visual query example. Let us assume the intention of a query is to retrieve lakes which are inside a particular country region. In Figure 1(a) the user just started to formulate the query. After he has specified that the type of the surrounding polygon $A$ should be a country, the type of the small polygon $B$ must be specified. As discussed above, a smart interface uses formal derivation processes for computing plausible candidates for object "type" specifications. For narrowing the set of possibilities we assume that two default rules are applicable: one saying that the interior small polygon $B$ could be a lake (Figure 1(b)) and another stating that $B$ could be a city (Figure 1(c)) if this does not lead to inconsistencies. Since an object can be either a lake or a city but not both, there is no way to believe in both possiblities at a time. This kind of default rule interaction is a simple example demonstrating the necessity of considering different possible worlds which must be maintained by the reasoning system. Depending on the default rule being used to conclude new knowledge, different subsequent conclusions might be possible.

Other potentially active default rules might be shown to produce inconsistencies with the set of current assertions without providing a possibility of using multiple worlds to avoid inconsistencies. For example, if there had been a default applied indicating that the small polygon B might as well be a country, we would have got a contradiction if we had an axiom (as part of our conceptual background knowledge) requiring that countries can never contain other countries. Thus, in our query context, the latter default cannot be applied and, as a consequence of computing and appropriately interpreting the set of possible worlds, we can compose a situation-adapted menu for the graphical user interface and the user can select between meaningful concepts for object B. In our specific example, the menu will contain items for lake and city but not for country. 


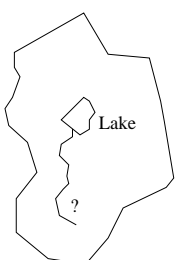

(a)

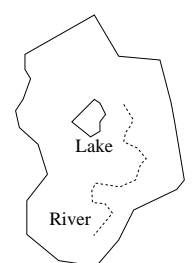

(b)

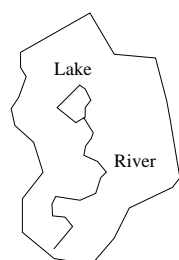

(c)

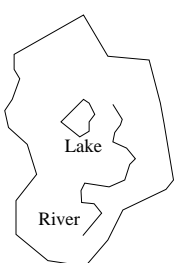

(d)

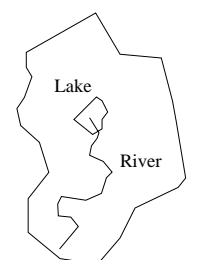

(e)

Fig. 2. Scenarios for situation-adapted completion of queries (see text).

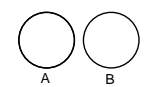

$\mathrm{dc}$

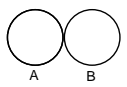

ec

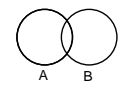

po

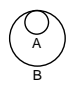

tpp

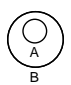

ntpp

Fig. 3. Elementary relations between two regions A and B. The inverses of TPP and NTPP as well as the relation EQ have been omitted.

If more than one possible world is computed, an intuitive criterion would be to select the world originating from a default with the more specific precondition or conclusion. E.g., in the query shown in Figure 2(a) we would prefer a default concluding that the thin graphical object might be a river_flowing_into_a_lake (which might be a useful concept in our scenario) instead of a "weaker" default concluding only that the object is an ordinary river.

The automatic augmentation of visual queries by conclusions of applied default rules can be seen as a specialization process. Therefore, this process might not only be useful during the construction of a visual query, but also useful as a tool for query refinement after a query has been executed that yields too many results. In addition, not only conceptual information is important. In a geographical information system context we also have to consider spatial relations between domain objects. An important example for spatial relations are topological relations. Due to its wide acceptance, we will rely on the well-known work about the RCC- 8 relations modeling topological relations between non-empty regular closed subsets in $R^{n}$ (see [4]). Figure 3 gives examples of the RCC-8 relations in the plane.

In the context of sketch-based visual querying, on the one hand it is sometimes useful to leave some spatial relations between graphical objects unspecified because they are unkwown or simply because the user is not willing to specify them. On the other hand, in order to actually draw a picture, the user must specifiy each spatial relation, even if it is just one of several possible (base) relations. The problem of how to specify "don't care relations" or "example relations" is well known and inherent in diagrammatic representations. It is similiar to the problem of visualizing visual disjunctions.

For example, in the query shown in Figure 2(b), we have a visual disconnected relation $(d c)$ between the river $^{1}$ and the lake. If we intended the river to be disjoint from the lake, the query answering system would not find any rivers

\footnotetext{
${ }^{1}$ Note that our river is assumed to be a thin twodimensional object.
} 
flowing into this lake. The problem is how can we specify that the river should be within the country (non-tangential proper part, ntpp) but leave the relation to the lake unspecified. As a possible solution to this problem, we could simply ignore each visible $d c$ relation. But, with this interpretation, we can now no longer state a query searching for rivers not flowing into this specific lake, which might be a very useful concept. We propose the following solution. For objects like the river that are drawn with a specific drawing attribute such as dashing, the universal spatial relation to other objects (disjunction of all base relations) is asserted. Dashed objects introduce no spatial query constraints. However, in some cases this would usually not match the users intention as there will be too many matches, i.e. the answer set will be too large. With the help of default knowledge we can automatically refine the query in a way that is appropriate according to the semantics of the objects involved in a query. So, we can guide the interpretation of spatial aspects by the help of conceptual background knowledge and application of defaults, yielding different hypotheses as possible worlds. A river flows into a lake or not, i.e. graphically both objects are either externally connected (relation $e c$, see also Figure 2(c)) or or they are disconnected (relation $d c$, see Figure $2(\mathrm{~d})$ ). With respect to a lake, there are no other possibilities. In our world model a river never overlaps with a lake (relation po, see also Figure 2(e)). This is assumed to be stated as an axiom as part of our general conceptual background knowledge. Besides defaults involving concept constraints we also have to take care of default rules with conclusions yielding new relation constraints.

The correct interpretation of the spatial relations explicit in a sketch depends on the conceptual background knowledge and demonstrates the benefits of integrated spatioterminological reasoning (see [6] for a first formal account on this topic). The important insight is the following duality: We can either use spatial relationships between object pairs to conclude their concept memberships, or we can use already known concept memberships to conclude particular spatial relationships between objects in the case of more general spatial relations (disjunctions of base relations). The conceptual background knowledge gives us the ability to conclude situation-specific candidates for spatial relationships. In this paper, a formalization for this inference process is presented. Based on this description logic language called $\mathcal{A L C R} \mathcal{P}\left(\mathcal{S}_{2}\right)$, which is briefly introduced, we discuss algorithms for computing so-called extensions ("possible worlds") of a world description and a set of defaults. We conclude with an application of spatioterminological default theory to problems in visual query systems and demonstrate the significance of the theory for spatioterminological reasoning in general and spatioterminological default reasoning in particular.

\section{Modeling Conceptual and Spatial Information}

We have seen the necessity for modeling conceptual background knowledge. The most widely accepted decidable formalisms with adequate expressiveness for this task are description logics. Basically, description logic formalisms distinguish 
between two kinds of building blocks: concepts and roles. Concepts denote sets of domain objects. Roles denote tuples of domain objects. As we have seen, in order to define meaningful concepts for spatial objects, it is also necessary to represent qualitative spatial relations and to exploit their various properties for reasoning. In particular, for a formalization of the motivating examples, we introduce a formalism for integrating reasoning about RCC-8 relations and reasoning about concepts. Since quantification over spatial relations is also needed for modeling (see below for examples), they should be represented as roles within a description logic formalism. In this section, we briefly introduce a description logic that supports this kind of modeling scheme. The logic is called $\mathcal{A L C R P}\left(\mathcal{S}_{2}\right)$ and is an instantiation of $\mathcal{A L C \mathcal { P }}(\mathcal{D})$ (see $[6,7]$ for an introduction). The name results from the well-known base language $\mathcal{A L C}(\mathcal{D})$ [1] and facilities for defining $R$ oles based on Predicates.

\subsection{Preliminaries}

Based on the facilities offered by $\mathcal{A L C R \mathcal { P }}\left(\mathcal{S}_{2}\right)$, roles representing RCC-8 relations can be defined and reasoned about using the formalism of "concrete domains" which provides an interface from a description logic reasoning system to another inference system possibly based on another theoretical background. The initial approach presented in [1] considered real numbers for engineering applications. The interface $\mathcal{D}$ is defined in terms of a pair of a domain $\Delta_{\mathcal{D}}$ and a set of names for predicates $P^{\mathcal{D}} \subseteq \Delta_{\mathcal{D}}^{\operatorname{arity}(P)}$. For integrating the description logic part of $\mathcal{A L C R \mathcal { P }}(\mathcal{D})$ (the abstract part) and the concrete part, the following admissibility criteria must hold. (1) The set of its predicate names must be closed under negation and must contain a name for a predicate concrete_domain_top for testing membership in $\Delta_{\mathcal{D}},(2)$ the satisfiability problem for finite conjunctions of predicates must be decidable. We briefly introduce the concrete domain $\mathcal{S}_{2}$ which can be used for representing two-dimensional spatial objects. Motivated by our introductory example we consider specific spatial objects whose spatial representations are given as polygons. $\mathcal{S}_{2}$ provides predicates that can be used to describe qualitative spatial RCC- 8 relationships as roles between spatial objects (see below for examples).

Definition 1. The concrete domain $\mathcal{S}_{2}$ is defined w.r.t. the topological space $\left\langle\mathbb{R}^{2}, 2^{\mathbb{R}^{2}}\right\rangle$. The domain $\Delta_{\mathcal{S}_{2}}$ contains all non-empty, regular closed subsets of $\mathbb{R}^{2}$ which are called regions for short. The set of predicate names is defined as follows:

- A unary concrete_domain_top predicate is-region with is-region $\mathcal{S}_{2}=\Delta_{\mathcal{S}_{2}}$ and its negation is-no-region with is-no-region ${ }^{\mathcal{S}_{2}}=\emptyset$.

- The 8 basic predicates dc, ec, po, tpp, ntpp, tppi, ntppi and eq correspond to the RCC-8 relations. Due to space restrictions we would like to refer to [7] for a formal definition of the semantics.

- In order to name disjunctions of base relations, we need additional predicates. Unique names for these "disjunction predicates" are enforced by imposing the 
following canonical order on the basic predicate names: dc, ec, po, tpp, ntpp, tppi, ntppi, eq. For each sequence $p_{1}, \ldots, p_{n}$ of basic predicates in canonical order $(n \geq 2)$, an additional predicate of arity 2 is defined. The predicate has the name $\mathrm{p}_{1}-\cdots-\mathrm{p}_{\mathrm{n}}$ and we have $\left(r_{1}, r_{2}\right) \in \mathrm{p}_{1}-\cdots-\mathrm{p}_{\mathrm{n}} \mathcal{S}_{2}$ iff $\left(r_{1}, r_{2}\right) \in \mathrm{p}_{1} \mathcal{S}_{2}$ or ... or $\left(r_{1}, r_{2}\right) \in \mathrm{p}_{\mathrm{n}}{ }^{\mathcal{S}_{2}}$. The predicate $\mathrm{dc}$-ec-po-tpp-ntpp-tppi-ntppi-eq is also called spatially-related.

- A binary predicate inconsistent-relation with inconsistent-relation $\mathcal{S}_{2}=\emptyset$ is the negation of spatially-related.

Proposition 1. $\mathcal{S}_{2}$ is admissible.

Proof. This is proven in [7]. Based on the results presented in [8] we can conclude that there exists always a model whose individuals are polygons which are not necessarily internally connected.

In the following we define the syntax and semantics of role and concept terms in $\mathcal{A L C R P}\left(\mathcal{S}_{2}\right)$.

Definition 2. Let $\mathrm{R}$ and $\mathrm{F}$ be disjoint sets of role and feature names, respectively. For brevity we also use the terms roles and features. Any element of $\mathrm{R} \cup \mathrm{F}$ is an atomic role term. A composition of features (written $f_{1} f_{2} \ldots$ ) is called a feature chain. A simple feature can be viewed as a feature chain of length 1. If $P$ is a predicate name from $\mathcal{S}_{2}$ with arity $n+m$ and $u_{1}, \ldots, u_{n}$ as well as $v_{1}, \ldots, v_{m}$ are feature chains, then the expression $\exists\left(u_{1}, \ldots, u_{n}\right)\left(v_{1}, \ldots, v_{m}\right) . P$ (role-forming predicate restriction) is a complex role term. Let $S$ be a role name and let $T$ be a role term. Then $S \doteq T$ is a terminological axiom.

Definition 3. Let $\mathrm{C}$ be a set of concept names which is disjoint to $\mathrm{R}$ and $\mathrm{F}$. Any element of $\mathrm{C}$ is a concept term (atomic concept term). If $C$ and $D$ are concept terms, $R$ is a role term, $P$ is a predicate name from $\mathcal{S}_{2}$ with arity $n$, and $u_{1}, \ldots, u_{n}$ are feature chains, then the following expressions are also concept terms: $C \sqcap D$ (conjunction), $C \sqcup D$ (disjunction), $\neg C$ (negation), $\exists R . C$ (exists restriction), $\forall R . C$ (value restriction), and $\exists u_{1}, \ldots, u_{n} . P$ (predicate exists restriction).

For all kinds of exists and value restrictions, the role term or the list of feature chains may be written in parentheses. Let $A$ be a concept name and let $D$ be a concept term. Then $A \doteq D$ and $A \sqsubseteq D$ are terminological axioms as well. A finite set of terminological axioms $\mathcal{T}$ is a terminology or TBox if no concept or role name in $\mathcal{T}$ appears more than once on the left hand side of a definition and, furthermore, if no cyclic definitions are present.

We now assign a meaning to $\mathcal{A L C \mathcal { R }} \mathcal{P}\left(\mathcal{S}_{2}\right)$ concept terms by giving a set-theoretic semantics as usual.

Definition 4. An interpretation $\mathcal{I}=\left(\Delta_{\mathcal{I}},{ }^{\mathcal{I}}\right)$ consists of a set $\Delta_{\mathcal{I}}$ (the abstract domain) and an interpretation function. ${ }^{\mathcal{I}}$. The sets $\Delta_{\mathcal{S}_{2}}$ and $\Delta_{\mathcal{I}}$ must be dis-

joint. The interpretation function maps each concept name $C$ to a subset $C^{\mathcal{I}}$ of $\Delta_{\mathcal{I}}$, each role name $R$ to a subset $R^{\mathcal{I}}$ of $\Delta_{\mathcal{I}} \times \Delta_{\mathcal{I}}$, and each feature name $f$ to 
a partial function $f^{\mathcal{I}}$ from $\Delta_{\mathcal{I}}$ to $\Delta_{\mathcal{S}_{2}} \cup \Delta_{\mathcal{I}}$, where $f^{\mathcal{I}}(a)=x$ will be written as $(a, x) \in f^{\mathcal{I}}$. If $u=f_{1} \cdots f_{n}$ is a feature chain, then $u^{\mathcal{I}}$ denotes the composition $f_{1}^{\mathcal{I}} \circ \cdots \circ f_{n}^{\mathcal{I}}$ of the partial functions $f_{1}^{\mathcal{I}}, \ldots, f_{n}^{\mathcal{I}}$. Let the symbols $C, D, R, P, u_{1}$, $\ldots, u_{m}$, and $v_{1}, \ldots, v_{m}$ be defined as in Definition 2 and 3 , respectively. Then the interpretation function can be extended to arbitrary concept and role terms as follows:

$$
\begin{array}{r}
(C \sqcap D)^{\mathcal{I}}:=C^{\mathcal{I}} \cap D^{\mathcal{I}},(C \sqcup D)^{\mathcal{I}}:=C^{\mathcal{I}} \cup D^{\mathcal{I}},(\neg C)^{\mathcal{I}}:=\Delta_{\mathcal{I}} \backslash C^{\mathcal{I}} \\
(\exists R . C)^{\mathcal{I}}:=\left\{a \in \Delta_{\mathcal{I}} \mid \exists b \in \Delta_{\mathcal{I}}:(a, b) \in R^{\mathcal{I}}, b \in C^{\mathcal{I}}\right\} \\
(\forall R . C)^{\mathcal{I}}:=\left\{a \in \Delta_{\mathcal{I}} \mid \forall b \in \Delta_{\mathcal{I}}:(a, b) \in R^{\mathcal{I}} \rightarrow b \in C^{\mathcal{I}}\right\} \\
\left(\exists u_{1}, \ldots, u_{n} . P\right)^{\mathcal{I}}:=\left\{a \in \Delta_{\mathcal{I}} \mid \exists x_{1}, \ldots, x_{n} \in \Delta_{\mathcal{S}_{2}}:\right. \\
\left.\left(a, x_{1}\right) \in u_{1}^{\mathcal{I}}, \ldots,\left(a, x_{n}\right) \in u_{n}^{\mathcal{I}},\left(x_{1}, \ldots, x_{n}\right) \in P^{\mathcal{S}_{2}}\right\} \\
\left(\exists\left(u_{1}, \ldots, u_{n}\right)\left(v_{1}, \ldots, v_{m}\right) \cdot P\right)^{\mathcal{I}}:=\left\{(a, b) \in \Delta_{\mathcal{I}} \times \Delta_{\mathcal{I}} \mid\right. \\
\exists x_{1}, \ldots, x_{n}, y_{1}, \ldots, y_{m} \in \Delta_{\mathcal{S}_{2}}: \\
\left(a, x_{1}\right) \in u_{1}^{\mathcal{I}}, \ldots,\left(a, x_{n}\right) \in u_{n}^{\mathcal{I}}, \\
\left(b, y_{1}\right) \in v_{1}^{\mathcal{I}}, \ldots,\left(b, y_{m}\right) \in v_{m}^{\mathcal{I}}, \\
\left.\left(x_{1}, \ldots, x_{n}, y_{1}, \ldots, y_{m}\right) \in P^{\mathcal{S}_{2}}\right\}
\end{array}
$$

An interpretation $\mathcal{I}$ is a model of a TBox $\mathcal{T}$ iff it satisfies $A^{\mathcal{I}}=D^{\mathcal{I}}\left(A^{\mathcal{I}} \subseteq D^{\mathcal{I}}\right)$ for all terminological axioms $A \doteq D(A \sqsubseteq D)$ in $\mathcal{T}$. A concept term $C$ is satisfiable w.r.t. a TBox $\mathcal{T}$ iff there exists a model $\mathcal{I}$ of $\mathcal{T}$ such that $C^{\mathcal{I}} \neq \emptyset$.

The following definition introduces the assertional language of $\operatorname{ALCR\mathcal {R}}\left(\mathcal{S}_{2}\right)$, which can be used to represent knowledge about individual worlds.

Definition 5. Let $O_{S_{2}}$ and $O_{A}$ be two disjoint sets of object names. If $C$ is a concept term, $R$ a role term, $f$ a feature name, $P$ a predicate name with arity $n$, $a$ and $b$ are elements of $O_{A}$ and $x$, and $x_{1}, \ldots, x_{n}$ are elements of $O_{S_{2}}$, then the following expressions are assertional axioms.

$$
a: C,(a, b): R,(a, x): f,\left(x_{1}, \ldots, x_{n}\right): P
$$

A finite set of assertional axioms is called ABox. An interpretation for the concept language can be extended to the assertional language by additionally mapping every object name from $O_{A}$ to a single element of $\Delta_{\mathcal{I}}$ and every object name from $\mathrm{O}_{S_{2}}$ to a single element from $\Delta_{\mathcal{S}_{2}}$. An interpretation satisfies an assertional axiom

$$
\begin{gathered}
a: C \text { iff } a^{\mathcal{I}} \in C^{\mathcal{I}}, \quad(a, b): R \text { iff }\left(a^{\mathcal{I}}, b^{\mathcal{I}}\right) \in R^{\mathcal{I}},(a, x): f \text { iff } f^{\mathcal{I}}\left(a^{\mathcal{I}}\right)=x^{\mathcal{I}}, \\
\left(x_{1}, \ldots, x_{n}\right): P \text { iff }\left(x_{1}^{\mathcal{I}}, \ldots, x_{n}^{\mathcal{I}}\right) \in P^{\mathcal{D}}
\end{gathered}
$$

An interpretation is a model of an $A B o x \mathcal{A}$ w.r.t. a TBox $\mathcal{T}$ iff it is a model of $\mathcal{T}$ and furthermore satisfies all assertional axioms in $\mathcal{A}$. An ABox is consistent w.r.t. a TBox $\mathcal{T}$ iff it has a model. 
Satisfiability of concept terms can be reduced to ABox consistency as follows: A concept term $C$ is satisfiable iff the $\operatorname{ABox}\{a: C\}$ is consistent. Another basic problem is to decide whether an assertional axiom $x$ is logically entailed by an $\operatorname{ABox} A, A \models x$, i.e. all models of $A$ are also models of $x$. If $x$ is an assertional axiom $i: C$, this is called the instance checking problem. If ABox consistency is decidable, instance checking can be reduced to checking whether $A \cup\{i: \neg C\}$ is inconsistent. We generalize the instance checking problem to the ABox entailment problem: An ABox $A$ logically entails an ABox $\left\{a_{1}, \ldots, a_{n}\right\}$, $A \models\left\{a_{1}, \ldots, a_{n}\right\}$ iff all models of $A$ are also models of the assertional axioms $a_{i}$. The ABox entailment problem can be reduced to checking whether $\forall i \in$ $\{1, \ldots, n\}: A \cup\left\{\neg a_{i}\right\}$ is inconsistent. However, in $\mathcal{A} \mathcal{L C} \mathcal{R} \mathcal{P}\left(\mathcal{S}_{2}\right)$, the negation of an assertional axiom is well-defined only for concept axioms: $\neg(i: C)$ iff $i: \neg C$. For default reasoning it is also necessary to check whether a role assertion $(a, b)$ : $\exists\left(u_{1} \ldots u_{n}\right)\left(v_{1} \ldots v_{m}\right) . P$ is logically entailed by an ABox $A$. This is discussed below.

The above-mentioned inference problems can be decided if $\mathcal{A} \mathcal{L C R} \mathcal{P}\left(\mathcal{S}_{2}\right)$, which is an instantiation of $\mathcal{A L C \mathcal { P }}(\mathcal{D})$, is decidable. In [6] it is shown that, unfortunately, the inference problem of checking the consistency of ABoxes in the "generic" language $\mathcal{A L C R \mathcal { P }}(\mathcal{D})$ is undecidable in general. However, in [7] a restricted variant of $\mathcal{A L C R P}(\mathcal{D})$ is described that is indeed decidable if only (syntactially) restricted concept terms are used.

Definition 6. A concept term $X$ is called restricted w.r.t. a TBox $\mathcal{T}$ iff its equivalent $X^{\prime}$ which is unfolded w.r.t. $\mathcal{T}$ and in negation normal form fulfills the following conditions: ${ }^{2}$

(1) For any subconcept term $C$ of $X$ ' that is of the form $\forall R_{1}$.D $\left(\exists R_{1} . D\right)$ where $R_{1}$ is a complex role term, $D$ does not contain any terms of the form $\exists R_{2} . E\left(\forall R_{2} . E\right)$ where $R_{2}$ is also a complex role term.

(2) For any subconcept term $C$ of $X$ ' that is of the form $\forall R . D$ or $\exists R . D$ where $R$ is a complex role term, $D$ contains only predicate exists restrictions that (i) quantify over attribute chains of length 1 and (ii) are not contained inside any value and exists restrictions that are also contained in $D$.

A terminology is called restricted iff all concept terms appearing on the righthand side of terminological axioms in $\mathcal{T}$ are restricted w.r.t. $\mathcal{T}$. An $A B$ ox $\mathcal{A}$ is called restricted w.r.t. a TBox $\mathcal{T}$ iff $\mathcal{T}$ is restricted and all concept terms used in $\mathcal{A}$ are restricted w.r.t. the terminology $\mathcal{T}$.

Proposition 2. The ABox consistency problem for restricted $\mathcal{A L C R \mathcal { P }}\left(\mathcal{S}_{2}\right)$ concept terms is decidable.

Proof. See Proposition 1 and [7].

\footnotetext{
${ }^{2}$ For technical reasons, we assume that a concept term is a subconcept term of itself. Any concept term can be transformed into an unfolded form by iteratively replacing concept and role names by their defining terms. An unfolded term is in negation normal form if negation is used only for concept names (for details see [7]).
} 
Proposition 3. The set of restricted $\mathcal{A L C R} \mathcal{P}\left(\mathcal{S}_{2}\right)$ concept terms is closed under negation.

Proof. See [7].

These results will be needed for the default reasoning algorithms dealing with $\mathcal{A L C R} \mathcal{P}\left(\mathcal{S}_{2}\right)$ concept and role terms (see below). The use of $\mathcal{A L C R} \mathcal{P}\left(\mathcal{S}_{2}\right)$ for spatioterminological domain modeling is demonstrated in the following sections. The examples discussed here provide a formalization of the examples used in the introduction and will subsequently be exploited to illustrate spatioterminological reasoning with defaults.

\subsection{Putting $\mathcal{A L C R} \mathcal{P}\left(\mathcal{S}_{2}\right)$ to Work}

Suppose we have the following $\mathcal{A L C R} \mathcal{P}\left(\mathcal{S}_{2}\right)$ TBox supplying our conceptual background knowledge. First, we define roles according to the spatial relationships needed in our application example. As an ontological decision we agree upon using the feature has_area for referring to the spatial representation of individuals.

$$
\begin{aligned}
\text { inside } & \doteq \exists(\text { has_area })(\text { has_area }) . t p p-n t p p \\
\text { contains } & \doteq \exists(\text { has_area })(\text { has_area }) . t p p i-n t p p i \\
\text { overlaps } & \doteq \exists(\text { has_area })(\text { has_area).po } \\
\text { touches } & \doteq \exists(\text { has_area })(\text { has_area }) . e c
\end{aligned}
$$

In addition, we give the definition of concepts required to model domain objects representing different kinds of regions in a TBox that satisfies the $\mathcal{A L C R} \mathcal{P}\left(\mathcal{S}_{2}\right)$ restrictedness criteria.

$$
\begin{aligned}
\text { area } & \doteq \exists \text { has_area.is-region } \\
\text { natural_region } & \doteq \neg \text { administrative_region } \\
\text { country_region } & \sqsubseteq \text { administrative_region } \sqcap \text { large_scale } \sqcap \text { area } \\
\text { city_region } & \sqsubseteq \text { administrative_region } \sqcap \neg \text { large_scale } \sqcap \text { area } \\
\text { lake_region } & \sqsubseteq \text { natural_region } \sqcap \text { area } \\
\text { river_region } & \sqsubseteq \text { natural_region } \sqcap \text { area }
\end{aligned}
$$

An area is a twodimensional region with some extent. Furthermore, we distinguish between administrative_regions and natural_regions which are disjoint concepts. The difference between a country_region and a city_region is that the former is large_scale, but the latter is not. Thus, these two concepts are disjoint as well. The intention behind the other concepts should be obvious. We would like to mention that these region concepts are basic concepts being used to define a set of concepts which are used by a query interface system (e.g. VISCO). For demonstration purposes we consider some of the concepts that might be used in a full-fledged (visual) query system.

$$
\begin{aligned}
& \text { country } \doteq \text { country_region } \sqcap \forall \text { contains. } \neg \text { country_region } \square \\
& \forall \text { overlaps. } \neg \text { country_region } \sqcap \forall \text { inside. } \neg \text { country_region } \\
& \text { city } \doteq \text { city_region } \sqcap \exists \text { inside.country_region } \\
& \text { lake } \sqsubseteq \text { lake_region } \\
& \text { river } \doteq \text { river_region } \sqcap \forall \text { overlaps. } \neg \text { lake_region } \sqcap \forall \text { inside. } \neg \text { lake_region }
\end{aligned}
$$


A country is a country_region and can never contain other country_regions. Also, countries never overlap other country_regions. Each city must belong to a specific country, i.e. must lie within a country. Unfortunately, we cannot write this directly as $\exists$ inside.country because the unfolded resulting term is no longer restricted. So, we have to use the somewhat weaker version with the base concept country_region. In our world model a city must be inside a country. For a river we require that it never overlaps or is inside with a lake_region.

$$
\text { river_flowing_into_a_lake } \doteq \text { river } \sqcap \exists \text { touches.lake_region }
$$

A river_flowing_into_lake is a specific river that touches a lake_region (please recall that the RCC- 8 relationships $e c$ and $p o$ and also $e c$ and ntpp-tpp are disjoint). It would be reasonable to state also that cities do not overlap other cities etc., but this is ignored here for the sake of brevity.

We have seen that $\mathcal{A} \mathcal{L C} \mathcal{R} \mathcal{P}\left(\mathcal{S}_{2}\right)$ provides the necessary expressiveness to model the objects in our geographic information system scenario. In the next section, these concepts will be augmented with defaults in order to demonstrate how the problems sketched in the introduction can be solved. In [7] more examples are given which also demonstrate the influence of spatial reasoning with RCC-8 relations on TBox reasoning (e.g. subsumption between concepts).

\section{Spatioterminological Reasoning with Defaults}

Let us now briefly review the theory about defaults and then show how to compute the different extensions of a closed terminological default theory in order to formalize the terms we already used informally.

\subsection{Preliminaries}

A default rule has the form

$$
\frac{\alpha: \beta_{1}, \beta_{2}, \ldots, \beta_{n}}{\gamma}
$$

where $\alpha, \beta_{i}$ and $\gamma$ are usually first-order formulae. Informally speaking, the idea behind these default rule is the following. $\alpha$ is called the precondition of the rule, the $\beta_{i}$ terms are called justifications, and $\gamma$ is the consequent. The formula $\gamma$ is added to the world description when $\alpha$ is entailed by the world description and each formula $\beta_{i}$ is consistent with the world description. In our case, $\alpha, \beta$ and $\gamma$ are not arbitrary first-oder formulae, but $\mathcal{A L C R \mathcal { P }}\left(\mathcal{S}_{2}\right)$ concept terms that fullfill the $\mathcal{A L C R P}\left(\mathcal{S}_{2}\right)$ restrictedness criteria. Because concept terms correspond to unary predicates ranging over a free variable, say $x$, these defaults are called open defaults. In contrast, closed defaults do not contain any free variables.

Using description logic terms in default rules instead of first-order or propositional logic formulae has first been considered in [2]. A terminological default theory is a pair $(A, D)$ where $A$ is an ABox, and $D$ is a finite set of terminological 
default rules that have to be closed over the ABox $A$. These closed default rules can be obtained by instantiating the free variable $x$ in the concept expressions with all explictly mentioned ABox individuals. Default rules are never applied to implicit individuals that might be introduced by $\exists$ restrictions. Due to this semantics, skolemization as originally proposed by Reiter to treat open defaults is not necessary (see [2] for a discussion of problems with skolemization).

\subsection{Solving the example problems}

Recalling our introductory example, let us define the following default rules:

$$
D_{1}=\frac{\text { area }: \text { city }}{\text { city }}, \quad D_{2}=\frac{\text { area }: \text { lake }}{\text { lake }}, \quad D_{3}=\frac{\text { area }: \text { country }}{\text { country }}
$$

Suppose we have an ABox $\{a:$ country, $b:$ area $,(a, b):$ contains, $(b, a):$ inside $\}$ corresponding to the visual query shown in Figure 1(a). Intuitively, answering the query means finding "equality assertions" that unify individuals in the query and in an ABox representing, for instance, a GIS database. Note that the unique name assumption does not hold for $\mathcal{A L C \mathcal { R }}\left(\mathcal{S}_{2}\right)$.

Closing defaults, i.e. instantiating the defaults $D_{1}, D_{2}, D_{3}$ over the ABox individuals $a$ and $b$ would yield 6 different closed defaults. Now, $\alpha, \beta$ and $\gamma$ have been replaced by the corresponding assertional axioms. We use the notation $D($ ind $)$ to refer to a default that is instantiated with the individual ind. Given our 6 closed default rules let us examine the status of each:

- Default $D_{1}(a)$ cannot be applied because adding $a$ : city to the ABox yields a contradiction with $a$ : country. country_region and city_region are disjoint concepts (due to large_scale and ᄀlarge_scale).

- Default $D_{1}(b)$ can be applied. Thus we can get an augmented ABox or extension one, corresponding to Figure 1(b):

$$
\{a: \text { country }, b: \text { city },(a, b): \text { contains },(b, a): \text { inside }\}
$$

- Default $D_{2}(a)$ cannot be applied, because adding $a$ : lake to the ABox yields a contradiction with $a$ : country, because a country is an administrative_region and a lake is defined as a natural_region, and both are disjoint concepts.

- Default $D_{2}(b)$ can be applied. Thus, we can get an augmented ABox or extension two, corresponding to Figure 1(c):

$$
\{a: \text { country }, b: \text { lake },(a, b): \text { contains },(b, a): \text { inside }\}
$$

However, if we have an ABox already augmented by the conclusion of default $D_{1}(b), b$ : city, we cannot apply $D_{2}(b)$. So, only one of $D_{1}(b)$ or $D_{2}(b)$ can be applied, resulting in two different extensions.

- Default $D_{3}(a)$ cannot be applied, because its conclusion is already entailed by the ABox. 


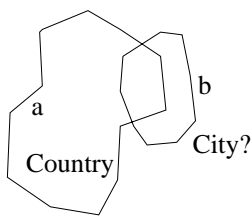

Can $b$ be a city?

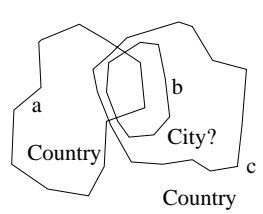

No!

Fig. 4. Subtle inferences due to geometric constraints.

- Default $D_{3}(b)$ cannot be applied even if no other default has been applied before. Adding the default's consequence $b$ : country would yield an inconsistent ABox because $a$ is already known to be a country and so, among others, $a: \forall$ contains. $\neg$ country_region holds. Because $(a, b)$ : contains holds and $b$ : country would imply $b$ : country_region, the default cannot be applied. Thus, we cannot get an extension corresponding to the wrong interpretation in Figure 1(d).

Another subtle inference can be demonstrated by showing that the default $D_{2}(b)$ cannot be applied to conclude that object $b$ in Figure 4 is a city. Trying to do so would result in a constraint $b:$ city $\sqcap$ ᄏinside.country_region. Therefore, polygon $a$ cannot be the appropriate country because $(b, a)$ : overlaps holds. Due to the exists restriction there exists an implicit individual $c$ which is a country_region such that $(b, c)$ : inside holds. As can be seen in Figure 4, there is no way to find a spatial arrangement such that $b$ is inside $c$ and $c$ does not overlap or contain $a$. Because $a$ is a country and, therefore, may not overlap or may not be contained in another country_region, there is no way to conlude that $b$ could possibly be a city.

\subsection{Computing Extensions}

Once we have a closed default theory, a set of consequences of such a theory is referred to as an extension which is a set of deductive closed formulae defined by a fixed point construction. We cite a formal definition taken from [2]. $T h(\Gamma)$ stands for the deductive closure of a set of formulae $\Gamma$. In a description logic context $\Gamma$ is an ABox.

Definition 7. Let $E$ be a set of closed formulae and $(A, D)$ be a closed default theory. We define $E_{0}:=A$ and for all $i \geq 0$

$E_{i+1}:=E_{i} \cup\left\{\gamma \mid \alpha: \beta_{1}, \ldots, \beta_{n} / \gamma \in D, \alpha \in T h\left(E_{i}\right), \neg \beta_{1}, \ldots, \neg \beta_{n} \notin T h(E)\right\}$.

Then, $T h(E)$ is an extension of $(A, D)$ iff

$$
T h(E)=\bigcup_{i=0}^{\infty} T h\left(E_{i}\right)
$$

In order to use this definition for computing extensions for default theories with $\mathcal{A L C R} \mathcal{P}\left(\mathcal{S}_{2}\right)$ concept and role terms, we extend the theory presented in [2]. 
Definition 8. A default $\alpha: \beta_{1} \ldots \beta_{n} / \gamma$ is called unfolded iff the concept and role terms in the $\alpha, \beta$ or $\gamma$ terms are in unfolded form.

In our case $\alpha, \beta_{i}$ and $\gamma$ may be ABoxes.

Definition 9. A closed default theory $(A, D)$ is called admissible iff for all assertional axioms $(x, y): \exists\left(u_{1} \ldots u_{n}\right)\left(v_{1} \ldots v_{m}\right) . P$ occurring in the $\alpha, \beta$ or $\gamma$ ABoxes of the unfolded defaults in $D$ it holds that $A \models x: \exists u_{1}$.concrete_domain_top, ... $A \models x: \exists u_{n}$.concrete_domain_top and $A \models y: \exists v_{1}$. concrete_domain_top, ... $A \models y: \exists v_{m}$.concrete_domain_top (see Definition 1 for concrete_domain_top in the case of $\mathcal{A L C R P}\left(\mathcal{S}_{2}\right)$ ).

Definition 10. An unfolded closed default $\alpha: \beta_{1} \ldots \beta_{n} / \gamma$ is called restricted iff all elements of $\alpha$ are either of the form $x: C$ with $C$ being a restricted $\mathcal{A L C R \mathcal { P }}\left(\mathcal{S}_{2}\right)$ concept term or of the form $(x, y): \exists\left(u_{1} \ldots u_{n}\right)\left(v_{1} \ldots v_{m}\right) . P{ }^{3}$

Theorem 1. The set of extensions of a closed $\mathcal{A L C R} \mathcal{P}\left(\mathcal{S}_{2}\right)$ default theory $(A, D)$ with all elements of $D$ being restricted can be effectively computed if $(A, D)$ is admissible.

Proof. Because the extension to be constructed is already used in each iteration step, Definition 7 cannot be directly transformed into a generator for the extensions but can be used as tester for determining whether a given ABox is an extension of a default theory $(A, D)$. The tester is applied to the powerset of the ABox $A \cup\left\{\gamma \mid \alpha: \beta_{1} \ldots \beta_{n} / \gamma \in D\right\}$. The test procedure induced by Definition 7 checks which of these ABoxes is an extension. The basic inference problem in this algorithm is to determine whether an assertional axiom $x$ is in the deductive closure of an ABox $\Gamma(x \in T h(\Gamma))$, i.e. is logically entailed by an ABox $\Gamma$ $(\Gamma \models x)$. In Section 2.1 we have already seen that in the case of assertional axioms of the form $x=i: C$ this can be reduced to checking whether $A \cup\{i: \neg C\}$ is inconsistent because the negation of a restricted concept yields a restricted concept term (see Proposition 3). The instance checking problem is decidable for restricted $\mathcal{A L C R} \mathcal{P}\left(\mathcal{S}_{2}\right)$ concepts terms (Proposition 2). The fixpoint can be constructed in a finite number of steps because we consider only a finite number of defaults. In the above definition, we are not only asking whether an ABox axiom is logically entailed by an ABox. We also have to decide the ABox equivalence problem: are the two ABoxes $A$ and $B$ equivalent, i.e. $T h(A)=T h(B)$. This is the case iff $A \models B$ and $B \models A$. Because each ABox is a set of ABox axioms, an ABox $B=\left\{b_{1}, b_{2}, \ldots, b_{n}\right\}$ follows from an ABox $A, A \models B$, iff $\forall b_{i} \in B: A \cup\left\{\neg b_{i}\right\}$ is inconsistent.

If $x$ is of the form $(a, b): R$ where $R$ is a role term, in general this technique cannot be applied because the negation of a role term might yield a concept term that cannot be expressed in $\mathcal{A L C R} \mathcal{P}\left(\mathcal{S}_{2}\right)$ (see also [7]). However, as Definition 10 states, considering assertional role axioms only axioms

\footnotetext{
${ }^{3}$ Role assertions of the form $(x, y): R$ with $R$ being a role name are not discussed here because we concentrate on spatioterminological reasoning problems which require complex role terms rather than role names.
} 
of the form $(x, y): \exists\left(u_{1} \ldots u_{n}\right)\left(v_{1} \ldots v_{m}\right) . P$ are possible in preconditions of defaults. According to the semantics of role exists restrictions (see Definition 4 ), $A \models(x, y): \exists\left(u_{1} \ldots u_{n}\right)\left(v_{1} \ldots v_{m}\right) . P$ holds iff $A \cup(x, y): \forall\left(u_{1} \ldots u_{n}\right)\left(v_{1} \ldots v_{m}\right) . \bar{P}$ is inconsistent. The $\mathcal{A L C R} \mathcal{P}\left(\mathcal{S}_{2}\right)$ language does not provide this kind of $\forall$ operator. However, we can safely replace this inconsistency test by checking whether $A \cup(x, y): \exists\left(u_{1} \ldots u_{n}\right)\left(v_{1} \ldots v_{m}\right) \cdot \bar{P}$ is inconsistent because the concrete domain and the default theory $(A, D)$ is required to be admissible (see Section alcrpdpreliminaries and Definition 9, respectively). This ensures that for all objects $x$, $y$ the fillers of the feature chains $u_{1} \ldots u_{n}$ and $v_{1} \ldots v_{m}$ are objects in $\mathrm{O}_{S_{2}}$ (see Definition 5). Because we have feature chains filled with concrete objects, we can safely use the $\exists$ operator rather than an $\forall$ operator (see the semantics in Definition 4).

We can easily check whether a default theory is admissible (see Definition 9) because the negation of concept exists restrictions is well-defined in $\mathcal{A L C R P}\left(\mathcal{S}_{2}\right)$. Note that the admissibility criterion for default theories is only required for checking whether $A \models \alpha$ iff $\alpha$ contains a complex role assertion. In contrast, when checking for all $i$ whether $A \forall \neg \beta_{i}$ holds, we have to decide whether $A \cup\left\{\beta_{i}\right\}$ is consistent. This completes the proof.

\subsection{Applying Restricted Default Theories}

We have already used spatial relationships to conclude possible concept memberships per default. A major achievement of our theory is that it is possible to conclude spatial relationsships between objects. Recalling our introductory example, we would like to be able to conclude that the allowed spatial relationsships between the river and the lake in Figure 2(b) are $e c$ (touches) and $d c$ (disconnected). These conclusions cannot be expressed with the limited terminological default rules introduced in [2] because there $\alpha, \beta$ and $\gamma$ are concept expressions. In [2], it is possible to conclude $\exists$ restrictions. For the river in our example, we could conclude $\exists$ touches.lake. But, of course, this does not require that the existing lake must coincide with the lake wich we specified in our graphical query. We therefore extended the terminological default rules by substituting the concept expressions $\alpha, \beta$ and $\gamma$ by so-called ABox patterns. These ABox patterns are basically ABoxes with placeholders for individuals. Closing the default rules instantiates the patterns with all possible combinations of individuals yielding ordinary $\mathcal{A} \mathcal{L C R} \mathcal{P}\left(\mathcal{S}_{2}\right)$ ABoxes. We can also refer to specific individuals (for instance, an individual lake such as "Bodensee"). It is quite obvious that every default rule consisting only of concept expressions can also be written as an ABox pattern default rule.

Returning to our example, we could, in principle, define a single default to conclude (lake,river) : $\exists($ has_area)(has_area).dc-ec, but if we want to reflect the default's conclusion at the user interface level, we must use two different defaults, concluding different RCC-8 base relations, corresponding to two different completions of the visual query:

$$
D_{1}=\frac{\{x: \text { lake }, y: \text { river },(x, y): \text { spatially_related }\}:\{(x, y): \text { disjoint }\}}{\{(x, y): \text { disjoint }\}},
$$




$$
D_{2}=\frac{\{x: \text { lake }, y: \text { river },(x, y): \text { spatially_related }\}:\{(x, y): \text { touches }\}}{\{(x, y): \text { touches }\}}
$$

Closing the patterns, i.e. instantiating $x, y$ over the ABox $A=\{l$ : lake, $r$ : river $\}$ would yield 8 different closed defaults. Note that, if we had $A=\{l$ : lake, $r$ : ᄀriver_flowing_into_a_lake, $r$ : river $\}$, the second default could not be applied.

\subsection{Terminological Default Reasoning with Specificity}

Of course, if it were already known that river is really a river_flowing_into_a_lake and we already specified a lake in our graphical query, we would like to conclude that the lake in the query should be the lake. If we specified more than one lake in our graphical query but only one river, different possibilities could be visualized and searched for. Note that this interpretation of the graphical query would not be possible without don't care relations.

$D_{3}=\frac{\{x: l a k e, y: \text { river_flowing_into_a_lake },(x, y): \text { spatially_related }\}:\{(x, y): \text { touches }\}}{\{(x, y): \text { touches }\}}$

In this case, we would like to render the application of $D_{1}$ and $D_{2}$ invalid, because they are "less specific" than $D_{3}$ (even if $D_{2}$ yields the same conclusion, touches). A default $D_{1}$ is said to be more specific than $D_{2}, D_{1} \prec D_{2}$ iff $\left(\alpha\left(D_{1}\right) \models\right.$ $\left.\alpha\left(D_{1}\right)\right) \wedge\left(\alpha\left(D_{2}\right) \not \models \alpha\left(D_{1}\right)\right)$ where $\alpha(D)$ denotes the precondition of the default $D$. The ABox entailment problem can be reduced to a number of ABox consistency checks (see above). Algorithms for computing the so-called $S$-extensions ( $S$ for specificity) have already been developed by Baader and Hollunder [3]. There is a strong conjecture that these algorithms can be applied in our admissible $\mathcal{A L C R} \mathcal{P}\left(\mathcal{S}_{2}\right)$ context as well. In contrast, the ordinary extensions are called $R$-extensions ( $R$ for Reiter). In our example, we would get two different $\mathrm{R}$ extensions, but only one S-extension containing the ABox axiom $(r, l)$ : touches. The other $R$-extension containing $(r, l)$ : disjoint could not be derived, since only the most specific active defaults are applied when computing extensions. This would render the application of $D_{1}$ and $D_{2}$ impossible because $D_{3}$ is also active and more specific than both $D_{1}$ and $D_{2}$.

\section{Conclusion}

To the best or our knowledge we have proposed a first theory for spatio-terminological default reasoning. Our new spatio-terminological default theory extends previous work done in [2] and [3]. The new contributions are: As a base language, the expressive spatio-terminological description logic $\mathcal{A L C R P}\left(\mathcal{S}_{2}\right)$ is used. Allowing not only concept terms as formulae occuring inside default rules but also $\mathcal{A L C R P}\left(\mathcal{S}_{2}\right)$ ABoxes with assertional role axioms is necessary from an application-oriented point of view but imposes a number of theoretical problems. We have shown that the possible extensions of a closed $\mathcal{A L C R} \mathcal{P}\left(\mathcal{S}_{2}\right)$ spatioterminological default theory can be effectively computed provided the ABoxes 
$A$ representing the world description satisifies an admissible criterion. As we have seen, the additional restrictions are not very tight. For the individuals involved in role assertional role axioms, we have to make sure in the world description that the concrete domain objects exist. Although the basic algorithm discussed in this paper might not be directly used in applications, there is a strong conjecture that the techniques used in the basic algorithm can also be used in the more efficent algorithms proposed in [2]. In order to make spatioterminological default resoning really practical this will be investigated in future work. We have demonstrated that interesting application problems concerning spatio-terminological default knowledge can be solved with the new theory.

\section{Acknowledgments}

We would like to thank Volker Haarslev, Carsten Lutz and Bernd Neumann for valuable discussions on the topics covered by this paper. Furthermore, thanks go to Anni-Yasmin Turhan and Omar Nuri for their work on the implementation of the $\operatorname{ALCRP}\left(\mathcal{S}_{2}\right)$ consistency checker and the default reasoning substrate, respectively. All deficiencies are due to our own faults, of course.

\section{References}

1. F. Baader and P. Hanschke. A scheme for integrating concrete domains into concept languages. In Twelfth International Conference on Artificial Intelligence, Darling Harbour, Sydney, Australia, Aug. 24-30, 1991, pages 452-457, August 1991.

2. F. Baader and B. Hollunder. Embedding defaults into terminological representation systems. J. Automated Reasoning, 14:149-180, 1995.

3. F. Baader and B. Hollunder. Priorities on defaults with prerequisites, and their application in treating specificity in terminological default logic. J. Automated Reasoning, 15:41-68, 1995.

4. A.G. Cohn, B. Bennett, J.M. Gooday, and N.M. Gotts. Representing and reasoning with qualitative spatial relations. In O. Stock, editor, Spatial and Temporal Reasoning, pages 97-134. Kluwer Academic Publishers, Dordrecht, 1997.

5. T. Cohn, L. Schubert, and S. Shapiro, editors. Proceedings of Sixth International Conference on Principles of Knowledge Representation and Reasoning (KR'98), Trento, Italy, June 2-5, 1998, June 1998.

6. V. Haarslev, C. Lutz, and R. Möller. Foundations of spatioterminological reasoning with description logics. In Cohn et al. [5], pages 112-123.

7. V. Haarslev, C. Lutz, and R. Möller. A description logic with concrete domains and a role-forming predicate operator. to appear in J. of Logic and Computation, 1999.

8. J. Renz. A canonical model of the region connection calculus. In Cohn et al. [5], pages 330-341.

9. M. Wessel and V. Haarslev. VISCO: Bringing visual spatial querying to reality. In 1998 IEEE Symposium on Visual Languages, Halifax, Canada, Sep. 1-4, pages 170-177. IEEE Computer Society Press, Los Alamitos, September 1998. 